\title{
Inflammatory syndromes including PIMS-TS in children hospitalised in the Malopolska region of Poland in the first wave of the COVID-19 pandemic
}

\author{
Lidia Stopyra ${ }^{1,2}$, Urszula Kania3 ${ }^{3}$, Przemko Kwinta ${ }^{3}$ \\ 'Stefan Żeromski Specialist Hospital in Krakow, Poland \\ ${ }^{2}$ Andrzej Frycz Modrzewski Krakow University, Krakow, Poland \\ ${ }^{3}$ Department of Pediatrics, Jagiellonian University Medical College, Krakow, Poland
}

\section{ABSTRACT}

Aim of the study: To describe cases meeting the criteria of Kawasaki disease (KD), with particular consideration of the first 2 cases of paediatric inflammatory multisystem syndrome temporarily associated with SARS-CoV-2 infection (PIMS-TS) in patients in the Malopolska region in the first wave of the disease caused by SARS-CoV-2 infection (COVID-19) pandemic.

Material and methods: From 4 March to 15 July 2020 a total of 11 children meeting KD criteria were hospitalised in the Department of Infectious Diseases and Paediatrics, S. Żeromski Specialist Hospital in Krakow and the Clinic of Children's Diseases, Department of Paediatrics, Jagiellonian University Medical College in Krakow. This paper analyses the data collected and describes in detail the disease in 2 children with PIMS-TS.

Results: None of the 11 children who met the criteria of KD had a positive SARS-CoV-2 PCR test on admission. SARS-CoV-2 IgG test results were positive in 2 patients. All children presented with fever, rash, and conjunctivitis; 9 with swollen hands and feet; 8 with mucous membrane changes; 5 with abdominal symptoms; 5 with respiratory symptoms; and 1 with neurological signs. As regards laboratory tests, C-reactive protein (CRP), procalcitonin, lymphocytes, platelets, All were assessed in all patients, albumin in 10/11, lactate dehydrogenase (LDH), fibrinogen in 9/11, troponin and ferritin in 6/11, pro-BNP, D-dimers in 8/11. Echocardiography was performed in all patients and showed coronary artery abnormalities in 4 children. In PIMS-TS significant elevation of procalcitonin, CRP, D-dimers, $N$-terminal prohormone of brain natriuretic peptide (NT-pro-BNP), and thrombocytopaenia were observed.

Conclusions:

1. Because of the asymptomatic course of SARS-CoV-2 infection in children who subsequently developed PIMS-TS and the absence of any viral genetic material in a sample collected from the upper airways upon PIMS-TS diagnosis, a detailed epidemiological interview and a reliable serology testing for SARS-CoV-2 infection are necessary in each case meeting KD or PIMS-TS criteria.

2. There is a need to look for new factors differentiating PIMS-TS and KD, other than polymerase chain reaction (PCR) and serology tests.

KEY WORDS:

children, coronavirus, Kawasaki syndrome, COVID-19, PIMS-TS.

\section{ADDRESS FOR CORRESPONDENCE:}

Lidia Stopyra, Stefan Żeromski Specialist Hospital in Krakow, Na Skarpie 67, 31-913 Krakow, Poland, e-mail: lidiastopyra@gmail.com 


\section{INTRODUCTION}

An increased incidence of severe pneumonia with respiratory failure was noted on 31 December 2019 in China in the region of Wuhan. On 9 January 2020, the Chinese authorities announced that the virus responsible for these infections had been identified as a zoonotic animal Betacoronavirus that was later re-named as SARS-CoV-2 (severe acute respiratory syndrome coronavirus 2) [1].

Fever, cough, and dyspnoea were initially indicated as the presenting symptoms of SARS-CoV-2 infection. Over time, however, other symptoms were added, such as: vomiting and diarrhoea, loss of smell and taste, myalgia, neurological symptoms, and rashes.

On 26 April 2020, the North Central London Clinical Commissioning Group and Paediatric Intensive Care Society informed about an increased incidence of a severe form of a paediatric inflammatory multisystem disease in the United Kingdom, possibly linked with SARS-CoV-2 infection [2]. The disease is an immune multisystem disorder and has been compared to Kawasaki disease (KD).

Kawasaki disease is an acute vasculitis, usually of a moderate grade, but also of a low or high grade. Its pathogenesis is not entirely clear, but immune mechanisms and a link with an infectious factor have been underlined [3, 4].

On 1 May 2020, the Royal College of Paediatrics and Child Health (RCPCH) published management guidelines for children with paediatric inflammatory multisystem syndrome temporally associated with SARS-CoV-2 infection (PIMS-TS) and proposed a case definition [5].

Moreover, other reports about PIMS-TS started appearing: from Italy [6], Canada [7], the USA [8], and France [9].

Despite an increasing number of reports about multisystem inflammatory disease associated with COVID-19, data regarding this disease entity are still scarce. The clinical picture resembles other childhood inflammatory diseases, such as Kawasaki disease (KD), toxic shock syndrome (TSS), or macrophage activation syndrome (MAS).

In comparison with patients with $\mathrm{KD}$, patients with PIMS-TS are in general older and with higher white blood cells, neutrophile count, C-reactive protein (CRP), fibrinogen, and troponin. They also tend to have deep lymphopaenia, anaemia, and low platelet counts $[6,10,11]$.

A significant portion of patients with PIMS-TS meet the criteria of $\mathrm{KD}$, but the inflammatory processes are more intense and markers of cardiac injury are higher [11].

\section{AIM OF THE STUDY}

The aim of this article is to describe the cases meeting criteria of the $\mathrm{KD}$, with particular consideration of the first 2 cases of PIMS-TS in patients in the Malopolska region in the first wave of the COVID-19 pandemic.

\section{MATERIAL AND METHODS}

Because of the COVID-19 pandemic announced by the World Health Organization (WHO) and the increasing incidence in Poland, the Department of Infectious Diseases and Paediatrics of S. Żeromski Specialist Hospital in Krakow was ordered by the province governor to treat children with COVID-19 and children with suspected COVID-19. The University Children's Hospital in Krakow was selected to provide highly specialist diagnosis and treatment if necessary, and a temporary COVID-19 unit was opened there as part of the Clinic of Children's Diseases. These organisational solutions centralised the care for children with suspected PIMS-TS in the Malopolska region. On 5 June 2020, the decision was made to create a register of PIMS-TS cases, in which all children with a diagnosis established after 4 March 2020 were entered retrospectively, and later other cases were added prospectively.

This article presents an analysis of the data gathered until 15 July 2020.

The following definitions were adopted:

Paediatric inflammatory multisystem syndrome temporarily associated with SARS-CoV-2 infection (PIMS-TS) meeting the criteria proposed by the WHO [12].

Children and adolescents $0-19$ years of age with fever $>3$ days and two of the following:

- rash or bilateral non-purulent conjunctivitis or muco-cutaneous inflammation signs (oral, hands, or feet),

- hypotension or shock,

- features of myocardial dysfunction, pericarditis, valvulitis, or coronary abnormalities (including ECHO findings or elevated troponin/N-terminal prohormone of brain natriuretic peptide [NT-proBNP]),

- evidence of coagulopathy (by prothrombin time [PT], partial thromboplastin time [PTT], elevated D-dimers),

- acute gastrointestinal problems (diarrhoea, vomiting, or abdominal pain),

and - elevated markers of inflammation such as erythrocyte sedimentation rate (ESR), CRP, or procalcitonin,

and - no other obvious microbial cause of inflammation, including bacterial sepsis, staphylococcal or streptococcal shock syndromes,

and - evidence of COVID-19 (RT-PCR, antigen test, or serology positive) or likely contact with patients with COVID-19.

Kawasaki disease was assessed in accordance with the criteria proposed by the American Heart Association (AHA) from 2017 with a division into a typical form (fever lasting longer than 5 days and the presence of at least 4 of the 5 following signs and symptoms: 1 ) bilateral conjunctivitis without exudate; 2) inflammation of the oral mucosa; 3) oedema, erythema of the hands and feet; 4) rash; 5) lymphadenopathy) and an atypical form (fever lasting longer than 5 days and the presence of 2 or 3 of the 5 signs and symptoms listed above) [13]. 


\begin{tabular}{|c|c|c|c|c|c|c|c|c|c|c|c|c|c|c|c|}
\hline जे & 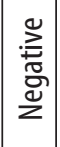 & 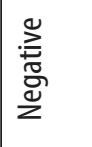 & & $\stackrel{m}{\wedge}$ & $\check{\varpi}$ & $\stackrel{\check{\nu}}{\check{\nu}}$ & $2=$ & $\stackrel{\check{\nu}}{\nu}$ & 28 & 2 & $\stackrel{2}{2}$ & 2 & 童 & & 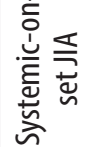 \\
\hline & 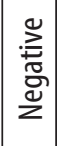 & 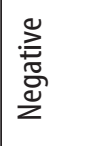 & & $\ln 7$ & $\stackrel{\check{z}}{\nearrow}$ & 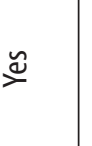 & $2=$ & $\stackrel{\breve{y}}{\nu}$ & 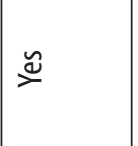 & 2 & 2 & & $\stackrel{2}{2}$ & & $\stackrel{2}{2}$ \\
\hline & 1 & 1 & & $\circ=$ & $\stackrel{\check{I}}{=}$ & $\stackrel{\check{\nu}}{\check{\nu}}$ & $2=$ & $\stackrel{\check{\varpi}}{\cong}$ & $\stackrel{\check{\nu}}{\simeq}$ & 2 & 2 & & 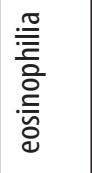 & : & 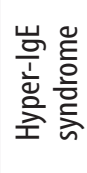 \\
\hline & 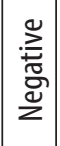 & 1 & & $0=$ & $\check{\overbrace{}}$ & 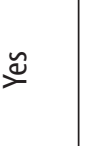 & $\stackrel{\mathscr{V}}{\chi}$ & $\stackrel{\check{\varpi}}{\searrow}$ & 2 & $\stackrel{2}{2}$ & $\stackrel{\check{\varpi}}{\searrow}$ & $\stackrel{\Xi}{\simeq}$ & $\begin{array}{l}\text { 总 } \\
\text { 言 }\end{array}$ & : & 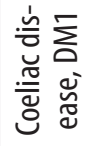 \\
\hline 商 & 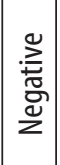 & 1 & & $n=$ & 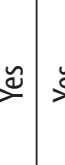 & 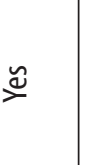 & $\stackrel{\check{g}}{\stackrel{2}{二}}$ & 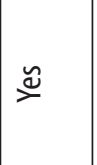 & $\frac{8}{2}$ & 2 & $\stackrel{\check{\nu}}{\rightleftharpoons}$ & $\cong$ & 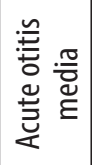 & $\mid$ & $\stackrel{2}{2}$ \\
\hline$\frac{1}{3}$ & 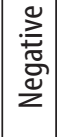 & 1 & & $\infty=$ & $\stackrel{\check{I}}{\check{I}}$ & 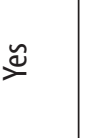 & $2=$ & $\stackrel{\check{\nu}}{ٍ}$ & 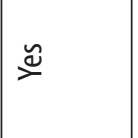 & $\frac{2}{2}$ & $\stackrel{0}{2}$ & 2 & 2 & $\mid$\begin{tabular}{|}
$\overline{\bar{\sigma}}$ \\
$\overline{\tilde{\xi}}$ \\
2 \\
2
\end{tabular} & 2 \\
\hline & 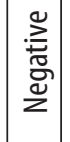 & 1 & & $=$ & $\stackrel{\check{\nu}}{\breve{~}}$ & 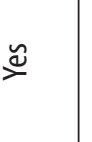 & $2=$ & $\stackrel{\check{y}}{\check{y}}$ & $\stackrel{\check{\nu}}{\check{\nu}}$ & 2 & $\stackrel{2}{2}$ & 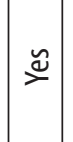 & 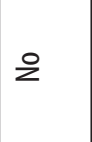 & 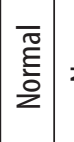 & $\stackrel{2}{2}$ \\
\hline zo & 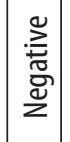 & 1 & & $\wedge$ & $\stackrel{\check{I}}{\simeq}$ & 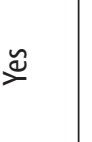 & $\stackrel{\mathscr{C}}{\not}=$ & $\stackrel{\check{\varpi}}{\check{\Sigma}}$ & $\stackrel{\check{\nu}}{\check{\nu}}$ & 2 & 2 & 2 & $\frac{8}{2}$ & $\mid \begin{array}{c}\overline{\bar{v}} \\
\overline{\bar{z}} \\
2\end{array}$ & $\stackrel{2}{2}$ \\
\hline & 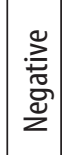 & $\begin{array}{l}\text { 䇏 } \\
\text { 离 }\end{array}$ & & $0=$ & 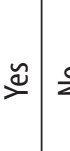 & 2 & $\stackrel{\square}{\varnothing}$ & 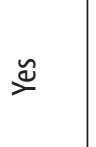 & $\stackrel{\check{\nu}}{\nu}$ & 2 & 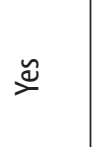 & $\stackrel{2}{2}$ & $\begin{array}{l}\text { 总 } \\
\text { 㲔 }\end{array}$ & $\mid \begin{array}{c}\overline{\mathrm{\sigma}} \\
\overline{\overline{\mathrm{E}}} \\
\overline{2}\end{array}$ & 2 \\
\hline & 总 & 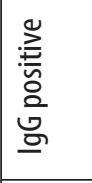 & & $n$ & 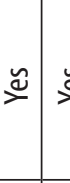 & 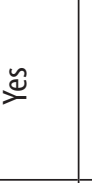 & $2=$ & $\stackrel{\check{\varpi}}{\check{\varpi}}$ & $\stackrel{\check{\nu}}{\beth}$ & $\stackrel{\Xi}{\simeq}$ & $\stackrel{\check{\varpi}}{\check{\Sigma}}$ & 2 & 2 & 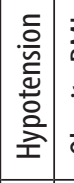 & 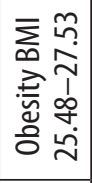 \\
\hline & 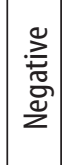 & 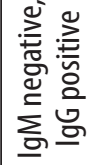 & & $0=$ & $\stackrel{\check{\Perp}}{\simeq}$ & $\stackrel{8}{2}$ & $\stackrel{\mathscr{a}}{\chi}$ & $\stackrel{\check{\varpi}}{\check{\varpi}}$ & $\stackrel{\check{\nu}}{\nu}$ & 2 & $\stackrel{\check{\varpi}}{\nsim}$ & & 2 & 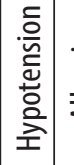 & 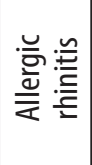 \\
\hline 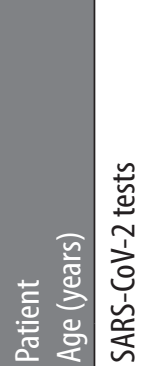 & 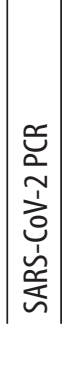 & 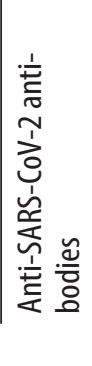 & 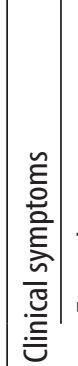 & 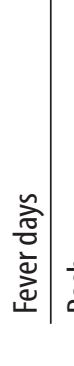 & 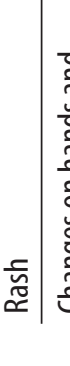 & 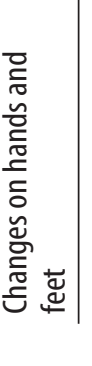 & 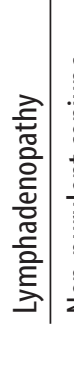 & 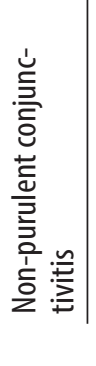 & 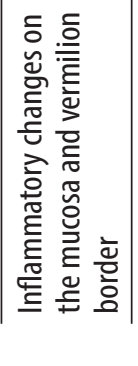 & 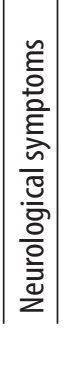 & 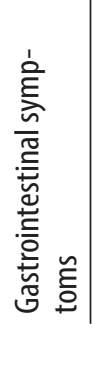 & 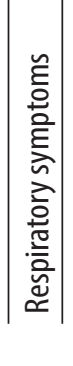 & 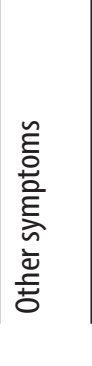 & & 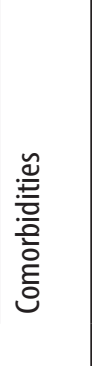 \\
\hline
\end{tabular}




\begin{tabular}{|c|c|c|c|c|c|c|c|c|c|c|c|c|c|c|c|c|c|c|c|c|}
\hline $\begin{array}{l}\hat{n} \\
\text { aे }\end{array}$ & ֻั & $\begin{array}{l}\infty \\
0 \\
0 \\
\end{array}$ & 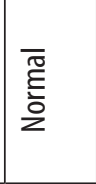 & 2 & ○े & $\stackrel{0}{2}$ & 2 & $\stackrel{0}{2}$ & 2 & $2 \mid \begin{array}{l}n \\
\tilde{n} \\
\tilde{n}\end{array}$ & 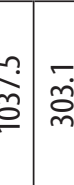 & $\stackrel{n}{n}$ & $\stackrel{m}{\sim}$ & 2 & 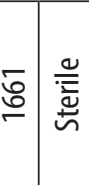 & & 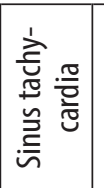 & 2 & 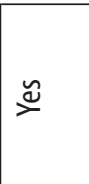 & 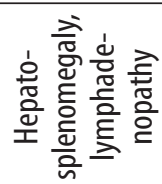 \\
\hline$\stackrel{n}{a} \sim$ & $\stackrel{8}{\stackrel{2}{r}}$ & 官 & 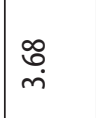 & 2 & 2 & $\frac{0}{2}$ & $\overline{\dot{o}}$ & $\stackrel{m}{\sim}$ & 2 & 2 & 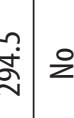 & 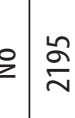 & $\begin{array}{l}F \\
\dot{n}\end{array}$ & $\stackrel{0}{2}$ & 导 & & 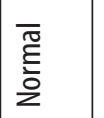 & 20 & 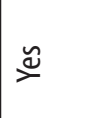 & $\begin{array}{l}\overline{\bar{g}} \\
\bar{z}\end{array}$ \\
\hline$\overline{\widehat{a}}_{\infty} \sim$ & 足 & \begin{tabular}{|l|}
0 \\
$\dot{\infty}$ \\
$\dot{o}$
\end{tabular} & $\grave{\mathscr{Y}}$ & 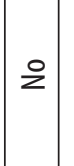 & ฉ̊ & $\stackrel{0}{2}$ & 2 & $\stackrel{0}{z}$ & 2 & 2 & $\begin{array}{lll} & \\
0 \\
0 \\
0\end{array}$ & 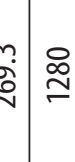 & $\stackrel{\circ}{m}$ & 1 & । & & 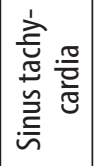 & $\overline{0}$ & 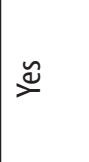 & 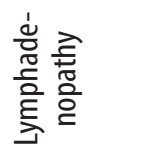 \\
\hline$\sum_{\substack{\infty \\
\infty}}^{n}$ & $\stackrel{\infty}{\stackrel{\infty}{0}}$ & $\underset{\infty}{+}$ & $\begin{array}{l}\overline{\widetilde{\pi}} \\
\stackrel{\underline{\xi}}{\partial} \\
z\end{array}$ & 2 & 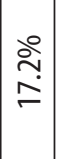 & 2 & $\stackrel{\infty}{\dot{0}}$ & 1 & $\stackrel{\tilde{g}}{\sigma}$ & $\approx$ & 12 & 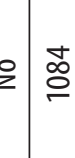 & $\stackrel{q}{+}$ & 2 & $\stackrel{0}{\underset{\tau}{\simeq}} 1$ & & \begin{tabular}{|l}
$\overline{\widetilde{g}}$ \\
हैं \\
$\dot{2}$
\end{tabular} & 2 & 2 & 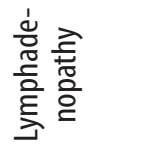 \\
\hline ટે & $\cong$ & $\approx$ & 胥 & 2 & 2 & 2 & in & $\stackrel{\infty}{\sim}$ & $\frac{0}{2}$ & $\begin{array}{lll}0 & 1\end{array}$ & 1 & ' & $\frac{0}{2}$ & 2 & \begin{tabular}{l|l}
$\underset{\Im}{\Im}$ & 1
\end{tabular} & & 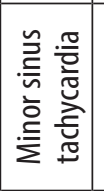 & 2 & 1 & 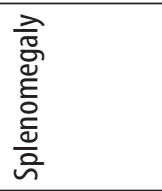 \\
\hline$\stackrel{2}{\sim}$ & fo & $\widehat{o}$ & 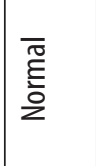 & 2 & 客 & 2 & 2 & $\stackrel{0}{2}$ & 2 & $\begin{array}{lll}0 & 1\end{array}$ & \begin{tabular}{l|l}
1 & 1
\end{tabular} & \begin{tabular}{l|l}
1 & 1
\end{tabular} & 1 & 1 & 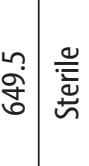 & & 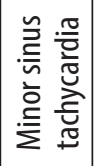 & 2 & $\frac{0}{2}$ & $\begin{array}{l}\overline{\bar{g}} \\
\stackrel{\bar{\xi}}{z}\end{array}$ \\
\hline$\stackrel{2}{a}$ & 苦 & $\stackrel{\circ}{=}$ & 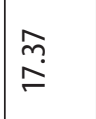 & $\frac{0}{2}$ & 2 & 2 & 家 & $\stackrel{n}{2}$ & 2 & $\begin{array}{lll}0 & 1\end{array}$ & $1 \quad 2$ & $\stackrel{2}{2} \hat{\tilde{m}}$ & 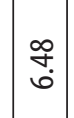 & I & 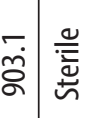 & & 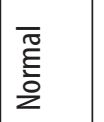 & 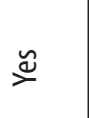 & 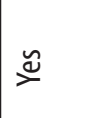 & 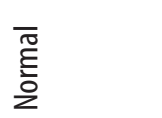 \\
\hline & $\bar{\sigma}$ & 足 & 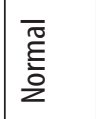 & $\stackrel{2}{2}$ & 客 & 2 & $\stackrel{2}{2}$ & 2 & $\frac{0}{2}$ & $\begin{array}{lll}0 & 1\end{array}$ & 12 & $\begin{array}{lll}0 & 1\end{array}$ & 1 & 1 & 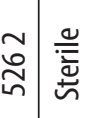 & & 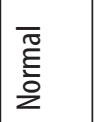 & $\stackrel{\Perp}{\Longleftarrow}$ & $\frac{0}{2}$ & $\begin{array}{l}\overline{\widetilde{\widetilde{g}}} \\
\overline{\hat{g}}\end{array}$ \\
\hline & & $\begin{array}{l}m \\
\dot{\theta}\end{array}$ & $\begin{array}{l}\overline{\widetilde{\sigma}} \\
\stackrel{\tilde{\xi}}{\bar{z}} \\
z\end{array}$ & 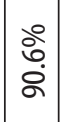 & 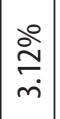 & 2 & in & $\stackrel{0}{2}$ & $\lesssim$ & 瓷完 & $\stackrel{2}{2} \stackrel{\infty}{m}$ & $\begin{array}{l}\infty \\
n\end{array}$ & ํ. & 1 & , & & $\begin{array}{l}\overline{\widetilde{\widetilde{g}}} \\
\frac{\bar{\partial}}{2}\end{array}$ & 2 & $\frac{0}{2}$ & 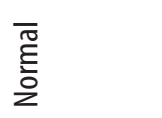 \\
\hline 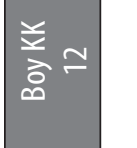 & 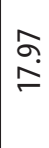 & @ & 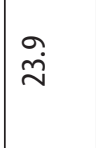 & ठ̊. & ஓे & ৪ & เ & $\stackrel{2}{\square}$ & 导 & 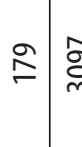 & ह్ & 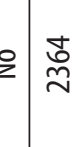 & ถู & 2 & 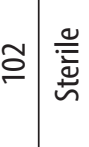 & & 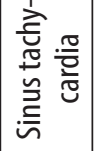 & $\stackrel{\check{\beth}}{\check{\nu}}$ & 2 & $\begin{array}{l}\overline{\widetilde{\Xi}} \\
\stackrel{\bar{\partial}}{z}\end{array}$ \\
\hline 䓂 & হু & $\bar{g}$ & $\bar{\sigma}$ & 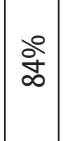 & 2 & 옴 & ธ & $\stackrel{\sim}{\sim}$ & $\frac{0}{2}$ & \begin{tabular}{l|l}
2 & \multicolumn{2}{c}{$\mid$}
\end{tabular} & $\stackrel{n}{m} \stackrel{2}{2}$ & 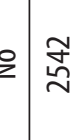 & $\begin{array}{l}\infty \\
0 \\
0 \\
0\end{array}$ & 2 & 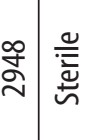 & 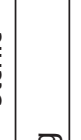 & 胥 & $\stackrel{\check{\beth}}{\check{\nu}}$ & $\stackrel{\Perp}{\Longleftarrow}$ & 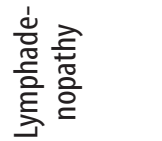 \\
\hline 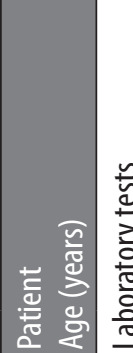 & 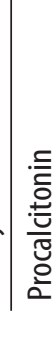 & 호 & 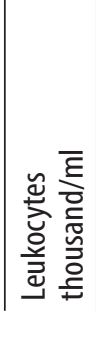 & 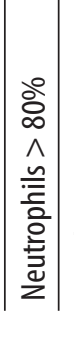 & 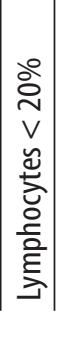 & 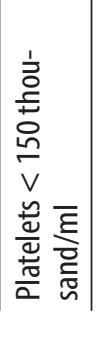 & 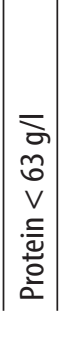 & 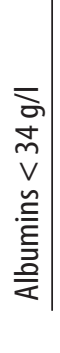 & 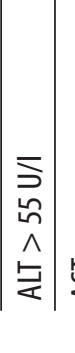 & 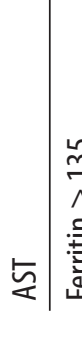 & 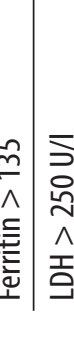 & 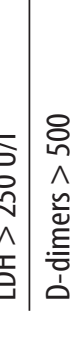 & 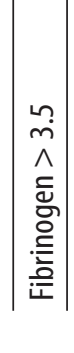 & 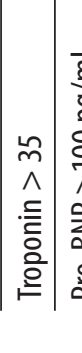 & 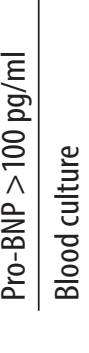 & 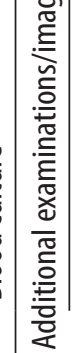 & |્ّ & 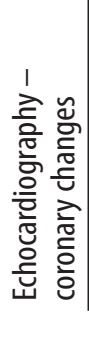 & 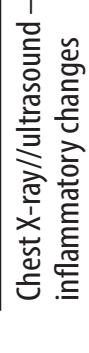 & 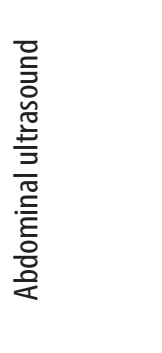 \\
\hline
\end{tabular}




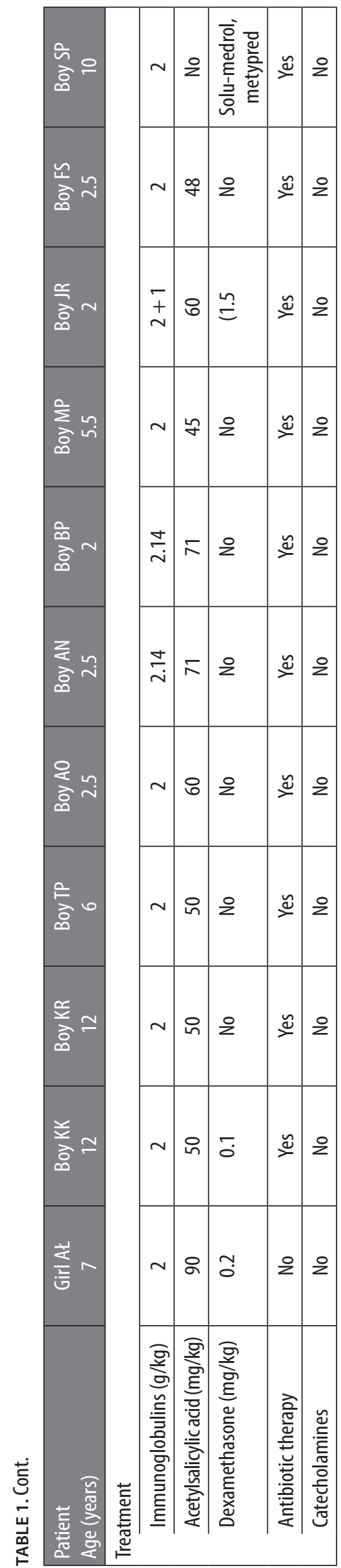

Kawasaki disease shock syndrome (KDSS) was defined as a case of Kawasaki disease with blood pressure decline of at least $20 \%$ of the due value or the presence of peripheral hypoperfusion [14].

Macrophage activation syndrome (MAS) was identified on the basis of the guidelines for MAS diagnosis in juvenile idiopathic arthritis issued by the Paediatric Rheumatology International Trials Organisation [15]. The above listed criteria are also used in the diagnostic process of other diseases, e.g. in Kawasaki disease [16].

The study was approved by the Ethics Committee of the Regional Medical Chamber in Krakow.

\section{RESULTS}

In the period from 4 March (i.e. from the date of the first case of SARS-CoV-2 infection in Poland) to 15 July, the Department of Infectious Diseases and Paediatrics and the Clinic of Children's Diseases treated 11 children who met the diagnostic criteria for KD. These were 10 boys and 1 girl aged $2-12$ years (mean age 5.8 years). Of these patients, 10 cases met criteria of typical KD and 1 with criteria for MAS. Hypotension was observed in 2 cases, but it did not meet the definition of KDSS. SARS$\mathrm{CoV}-2$ infection was confirmed in 2 cases. In the first of these cases, the typical symptoms of Kawasaki disease appeared nearly 4 weeks after the confirmed infection. The boy was earlier treated on an outpatient basis. The PCR test performed after nasopharyngeal sample collection at admission and after day 1 revealed no SARSCoV-2 infection, and the boy was considered a convalescent. The presence of IgG antibodies against SARSCoV-2 were detected using the ELISA assay. In the second case, the interview indicated COVID-19 approximately 4 weeks earlier, but the child did not have a nasopharyngeal swab for SARS-CoV-2 taken then. Upon admission to the Department of Infectious Diseases and Paediatrics, the swab test showed no SARS-CoV-2, but anti-SARSCoV-2 IgG antibodies were detected in the ELISA assay. In the remaining cases, SARS-CoV-2 infection was not confirmed.

Table 1 presents detailed patient characteristics.

A detailed description of the 2 children with confirmed SARS-CoV-2 infection is presented below.

\section{CASE 1}

A 12-year-old boy was diagnosed with SARS-CoV-2 infection on 28 May 2020 based on a positive PCR test result and was treated on an outpatient basis. The course of infection was asymptomatic, and the boy stayed at home in isolation. He reported to the Department of Infectious Diseases and Paediatrics on 24 June due to high fever (39-40 degrees) persisting for 1 day and diarrhoea. The last SARS-CoV-2 PCR test performed on 17 June was positive. The boy had presented no comorbidities 
apart from obesity (BMI $-25.5 \mathrm{~kg} / \mathrm{m}^{2}$, $>97^{\text {th }}$ centile). Physical examination conducted at admission revealed no significant irregularities. Laboratory findings were as follows: high CRP (125 mg\%, IQR < 5), elevated leukocytes $(19,400 / \mathrm{ml}, \mathrm{N} 4500-13,000)$, lymphocytopaenia (12\% IQR 15-45), platelet count (242,000/ml, IQR 180,000430,000), and ALT (121 IU/ml IQR 0-55). Stool examination revealed no Ag of rota- or adenovirus, or Campylobacter. A sample of stool for bacteriological examination was taken. Chest X-ray and abdominal ultrasound were normal. Two consecutive PCR tests of the nasopharyngeal swab showed no signs of SARS-CoV-2, and, in accordance with the guidelines of the WHO and the Polish Chief Sanitary Inspectorate, the boy was considered a convalescent.

On day 2 of hospital treatment, the patient developed petechial rash on the feet and non-purulent conjunctivitis and cough. On the subsequent days, the patient's general condition worsened, high fever and diarrhoea persisted. According to ESPGHAN and ESPID 2014 Guidelines for the Management of Acute Gastroenteritis in children in Europe [17] azithromycin was ordered. In the subsequent days the boy developed oedema of the hands and feet, inflammatory changes on the pharynx, mucosa, and vermilion border. Additional tests revealed increasing inflammatory markers and elevated transaminase activity. Azithromycin was discontinued and ceftriaxone with vancomycin was started. The bacteriological examinations of blood, stool, and urine were negative. Serology tests for HAV, HBV, HCV, HIV, EBV, and CMV were all negative. A decreased vitamin $\mathrm{D}_{3}$ level was noted. Laboratory findings were as follows: high CRP $(190 \mathrm{mg} \%$, IQR $<5)$, high procalcitonin $(17.97 \% \mathrm{IQR}<0.05)$, fibrinogen (6.5 g/l, IQR 1.8-3.5), ferritin (3095, N 21-275 ng/ml), elevated leukocytes $(23,900 / \mathrm{ml}, \mathrm{N} 4500-13,000)$, lym-

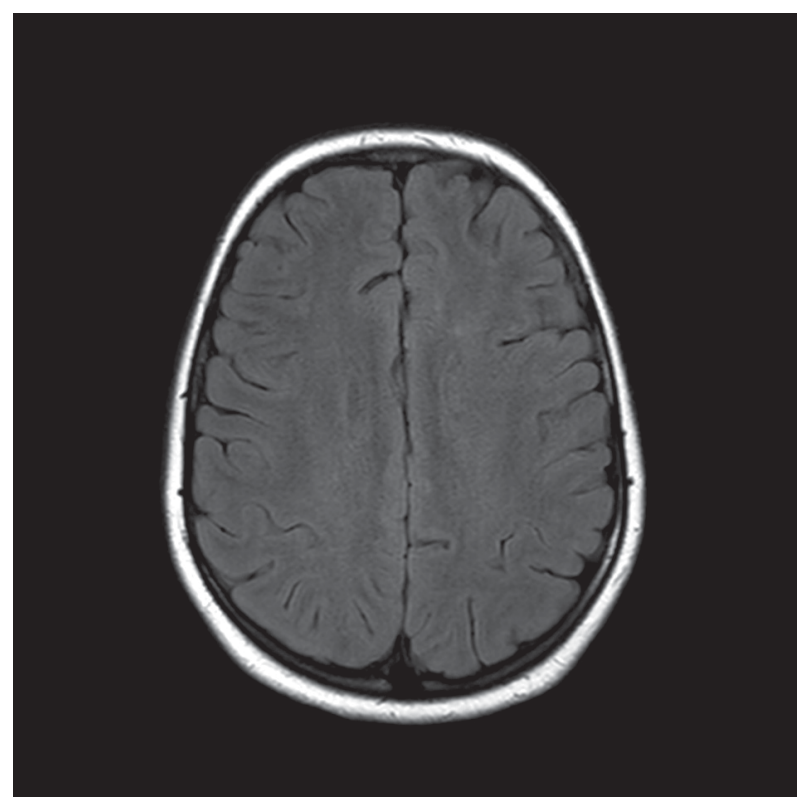

FIGURE 1. Boy KK - non-specific changes in the frontal lobes-1 phocytopaenia (4\%, IQR 15-45), thrombocytopaenia (90,000/ml, IQR 180,000-430,000), ALT (240 IU/ml, IQR 0-55), and hypoalbuminaemia (24 g/l, IQR 35-50). The presence of IgG antibodies against SARS-CoV-2 was confirmed using an ELISA assay. After 5 days of continuing fever, PIMS-TS with KD-like presentation was diagnosed. According to AHA and SHARE guidelines and the RAISE findings, immunoglobulin infusion, acetylsalicylic acid, and dexamethasone were started (Kobayashi score 8 points, high-risk patient with acute KD) [13, $18,19]$. The patient's condition rapidly improved: fever subsided, inflammatory markers decreased, but oedema appeared and blood pressure dropped to $80 / 60 \mathrm{~mm} \mathrm{Hg}$. Troponin was increasing. ECG and echocardiography were normal. Owing to reported visual hallucinations and periodical confusion, a brain MRI was ordered which revealed non-specific demyelinating lesions in the left frontal lobe (Figure 1). The diagnosis was extended to include EEG, which demonstrated discrete temporo-occipital and central lesions. All neurological symptoms subsided 4 days after treatment initiation.

The follow-up echocardiography conducted 6 days after the diagnosis revealed moderate, non-uniform coronary artery dilatation.

\section{CASE 2}

A 7-year-old girl was admitted on day 6 of a disease manifesting with high fever, rash, non-purulent conjunctivitis, lymphadenopathy, oedema, and erythema of the hands and feet, as well as inflammatory changes on the pharynx and oral mucosa. The interview revealed long-term and close contact with a person infected with SARS-CoV-2. Additional tests showed high values of inflammatory markers - procalcitonin (14.91\%, IQR

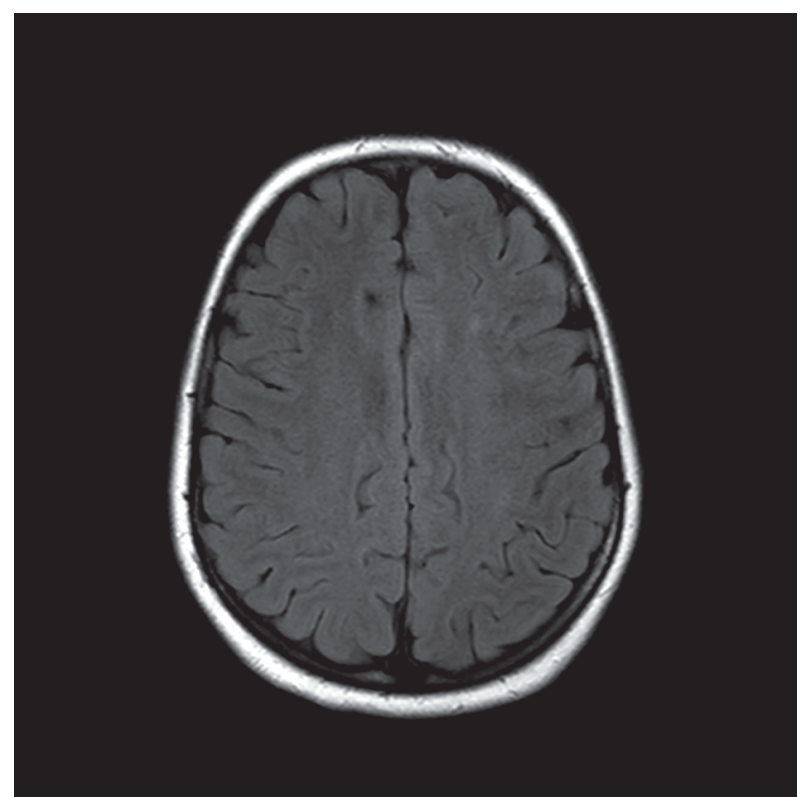

FIGURE 2. Boy KK - non-specific changes in the frontal lobes-2 
$<0.05)$, CRP $(109.1 \mathrm{mg} \%$, IQR < 5), leukocytes $(16,100 /$ ml, IQR 4500-13,000), neutrophiles 84\% (IQR 40-60), fibrinogen (6.08 g/l, IQR 1.8-3.5), decreased platelet count $(164,000 / \mathrm{ml}$, IQR $180,000-430,000)$, and elevated D-dimers (2542 ng/ml, IQR 0-500). Furthermore, lung ultrasound demonstrated inflammatory consolidations, and abdominal ultrasound revealed lymphadenopathy. SARS-CoV-2 IgG antibodies were confirmed using the ELISA assay.

PIMS-TS with KD-like presentation was diagnosed.

According to AHA and SHARE guidelines and the RAISE findings, immunoglobulin infusion, acetylsalicylic acid, and dexamethasone were started (Kobayashi score 4 points) $[13,18,19]$. There was a rapid improvement - fever subsided and inflammatory markers decreased.

Echocardiography showed coronary artery thickening with no pathological dilatations.

\section{DISCUSSION}

For the past 2 decades, coronaviruses have been taken into account as factors that might play a role in the pathogenesis of Kawasaki disease. In 2005 in New Haven, New Haven Coronavirus (HCoV-NH) was detected in the material collected from the airways of 8 of 11 patients with Kawasaki disease [20].

However, in response to this report, a Japanese study [21] demonstrated RNA of HCoV-NH in $2 \%$ of 208 children with upper respiratory tract infection and in none of 19 children diagnosed with Kawasaki disease in the same time period.

Another group of Japanese authors examined the prevalence of Kawasaki disease in the course of infections caused by $\mathrm{HCoV}-229 \mathrm{E}$ coronavirus using immunofluorescence assays [21]. The prevalence of anti$\mathrm{HCoV}-229 \mathrm{E}$ antibodies was much greater in patients with Kawasaki disease than in controls [22].

During the COVID-19 pandemic, reports of cases that meet the criteria of typical or atypical Kawasaki disease have been arriving from countries with a high incidence of infection (United Kingdom, Italy, USA, France). Attempts have been made to show a relationship between these reports and the SARS-CoV-2 infection.

This demonstrates that coronaviruses may indeed be a significant factor in the pathogenesis of Kawasaki disease, and that SARS-CoV-2, a particularly virulent strain, is responsible for the occurrence of PIMS-TS in genetically predisposed individuals.

Currently PIMS-TS has several definitions developed by the RCPCH [5], CDC [23], and WHO [12].

PIMS-TS is a rare disease. The literature states that it affects only 1 per 1000 children infected with SARSCoV-2 [6]. That is why all reports, and especially those from countries with low COVID-19 incidence, have limitations associated with a low number of reported cases.
Both patients with a confirmed SARS-CoV-2 infection hospitalised in the Malopolska region presented with gastrointestinal signs and symptoms, such as vomiting and diarrhoea. Reports from Italy [6], France [9], Austria [1], the United Kingdom [1], and the USA [10] are similar in this respect. As in the previous reports, SARS-CoV-2confirmed PIMS-TS patients had high inflammatory markers (procalcitonin, CRP, fibrinogen) and low platelet count. Cardiovascular involvement was confirmed in both the cases from the Malopolska region (elevated troponin, NT pro-BNP, involvement of the coronary arteries). In other reports, cardiovascular involvement is also more frequently described for PIMS-TS patients with confirmed SARS-CoV-2 infection than in KD patients $[1,6,7,8,10]$.

Amongst irregularities found in laboratory tests, attention should be drawn to a low vitamin $\mathrm{D}_{3}$ concentration $(9.3 \mathrm{ng} / \mathrm{ml}$ IGR $30-50)$ in a patient with a severe course of PIMS-TS and a confirmed SARS-CoV-2 infection. There is vast evidence that vitamin $\mathrm{D}$ deficiency may lead to endothelial dysfunction and pathological changes in the vascular system [24], which is of significance in the pathogenesis of $\mathrm{KD}$ and may be a potential risk factor for a more severe course of COVID-19 [25], as in the case reported above.

In both children with KD and confirmed SARSCoV-2 infection, the infection was asymptomatic, and the symptoms of PIMS-TS developed approximately 4 weeks after the onset of infection. In these 2 cases, the genetic material of SARS-CoV-2 was not found in the upper airway swabs at the onset of the PIMS-TS symptoms, but the presence of anti-SARS-CoV-2 antibodies in the serum was confirmed. This is the case for the majority of the reported cases in the world [1]. Considering the pathogenesis of PIMS-TS, it seems that the disease symptoms may develop when the virus is already absent in the material collected from the upper airways, and in these cases serology testing is necessary to confirm a relationship between SARS-CoV-2 infection and PIMS-TS.

Amongst the differences between the previously reported cases of PIMS-TS in the world and PIMS-TS diagnosed in children with a confirmed SARS-CoV-2 infection from the Malopolska region, the following aspects should be highlighted:

Psychiatric symptoms that developed in the boy KK in the form of visual hallucinations and altered consciousness presenting as clear confusion. To date, the neurological signs and symptoms reported in the course of PIMS-TS have included discrete meningeal signs with accompanying CSF cytosis $[9,26]$ or have not been specified [10]. MRI revealed non-specific demyelinating changes. In one report, hemosiderosis-like changes were reported on the MRI of a child with PIMS-TS [26]. In the case of KK, MRI showed non-specific changes in the frontal lobes to be differentiated between demyelinating 
lesions and lesions detected in dementia syndromes. The child had no neurological disorders in his family history. The neurological signs appeared during IVIG treatment. Psychiatric symptoms, visual hallucinations, and altered consciousness are not described as IVIG treatment adverse events. Thromboembolic complications are described as such, but an MRI done at that time did not show any changes typical for thromboembolic complications. These symptoms and MRI changes in PIMS TS warrant further observation.

A relatively mild course. The patients from the Malopolska region were hospitalised at the department of paediatric infectious diseases. They did not require intensive care, mechanical ventilation, or catecholamine administration.

Rapid improvement following IVIG. All symptoms regressed and inflammatory markers decreased in both reported cases of PIMS-TS with confirmed SARS-CoV-2 infection. The previous literature draws attention to the inefficacy of IVIG in PIMS-TS $[6,9,10]$. In both PIMS TS cases with a confirmed SARS-CoV-2 infection, IVIG was initiated immediately after the 5 th day of fever. It should be mentioned that the WHO guidelines for PIMS-TS diagnosis include fever lasting more than 3 days [12].

\section{CONCLUSIONS}

Because of the asymptomatic course of SARS-CoV-2 infection in children who subsequently developed PIMSTS and the absence of any viral genetic material in a sample collected from the upper airways at PIMS-TS diagnosis, the current epidemiological situation warrants a detailed epidemiological interview and reliable serology testing for SARS-CoV-2 infection in each patient meeting KD or PIMS-TS criteria.

It is necessary to look for new factors differentiating PIMS-TS and KD, other than PCR and serology tests.

\section{DISCLOSURE}

The authors declare no conflict of interest.

\section{REFERENCES}

1. European Centre for Disease Prevention and Control. Paediatric inflammatory multisystem syndrome and SARS-CoV-2 infection in children. Stockholm: ECDC, 2020.

2. Mahase E. Covid-19: concerns grow over inflammatory syndrome emerging in children. BMJ 2020; 369: m1710.

3. Rowley AH. Is Kawasaki disease an infectious disorder? Int J Rheum Dis 2018; 21: 20-25.

4. Jordan-Villegas A, Chang ML, Ramilo O, Mejías A. Concomitant respiratory viral infections in children with Kawasaki disease. Pediatr Infect Dis J 2010; 29: 770-772.

5. Royal College of Paediatrics and Child Health. Paediatric multisystem inflammatory syndrome temporally associated with COVID-19. UK: 2020.
6. Verdoni L, Mazza A, Gervasoni A, et al. An outbreak of severe Kawasaki-like disease at the Italian epicentre of the SARS-CoV-2 epidemic: an observational cohort study. Lancet 2020; 395: 1771-1778.

7. McCrindle BW, MD, Manlhiot C. PhD2. SARS-CoV-2-Related Inflammatory Multisystem Syndrome in Children. JAMA 2020; 324: 246-248.

8. Jones VG, Mills M, Suarez D, et al. COVID-19 and Kawasaki Disease: Novel Virus and Novel Case. Hosp Pediatr 2020; 10: 537-540.

9. Toubiana J, Poirault C, Corsia A, et al. Kawasaki-like multisystem inflammatory syndrome in children during the covid-19 pandemic in Paris, France: prospective observational study. BMJ 2020; 369: $\mathrm{m} 2094$.

10. Feldstein LR, Rose EB, Horwitz SM, et al.; Overcoming COVID-19 Investigators; CDC COVID-19 Response Team. Multisystem Inflammatory Syndrome in U.S. Children and Adolescents. N Engl J Med 2020; 383: 334-346.

11. Whittaker E, Bamford A, Kenny J, et al.; PIMS-TS Study Group and EUCLIDS and PERFORM Consortia. Clinical Characteristics of 58 Children With a Pediatric Inflammatory Multisystem Syndrome Temporally Associated With SARS-CoV-2. JAMA 2020; 324: 259-269.

12. WHO. Multisystem inflammatory syndrome in children. 2020.

13. McCrindle BW, Rowley AH, Newburger JW, et al.; American Heart Association Rheumatic Fever, Endocarditis, and Kawasaki Disease Committee of the Council on Cardiovascular Disease in the Young; Council on Cardiovascular and Stroke Nursing; Council on Cardiovascular Surgery and Anesthesia; and Council on Epidemiology and Prevention. Diagnosis, Treatment, and Long-Term Management of Kawasaki Disease: A Scientific Statement for Health Professionals From the American Heart Association. Circulation 2017; 135: e927-e999.

14. Kanegaye JT, Wilder MS, Molkara D, et al. Recognition of a Kawasaki disease shock syndrome. Pediatrics 2009; 123: e783-789.

15. Ravelli A, Minoia F, Davì S, et al.; Paediatric Rheumatology International Trials Organisation; Childhood Arthritis and Rheumatology Research Alliance; Pediatric Rheumatology Collaborative Study Group; Histiocyte Society. 2016 Classification Criteria for Macrophage Activation Syndrome Complicating Systemic Juvenile Idiopathic Arthritis: A European League Against Rheumatism/ American College of Rheumatology/Paediatric Rheumatology International Trials Organisation Collaborative Initiative. Arthritis Rheumatol 2016; 68: 566-576.

16. García-Pavón S, Yamazaki-Nakashimada MA, Báez M, et al. Kawasaki Disease Complicated With Macrophage Activation Syndrome: A Systematic Review. J Pediatr Hematol Oncol 2017; 39: 445-451.

17. Guarino A, Ashkenazi S, Gendrel D, et al.; European Society for Pediatric Gastroenterology, Hepatology, and Nutrition; European Society for Pediatric Infectious Diseases. European Society for Pediatric Gastroenterology, Hepatology, and Nutrition/European Society for Pediatric Infectious Diseases evidence-based guidelines for the management of acute gastroenteritis in children in Europe: update 2014. J Pediatr Gastroenterol Nutr 2014; 59: 132-152.

18. de Graeff N, Groot N, Ozen S, et al. European consensus-based recommendations for the diagnosis and treatment of Kawasaki disease the SHARE initiative. Rheumatology (Oxford) 2019; 58: 672-682.

19. Kobayashi T, Inoue $Y$, Takeuchi $K$, et al. Prediction of intravenous immunoglobulin unresponsiveness in patients with Kawasaki disease. Circulation 2006; 113: 2606-2612.

20. Esper F, Shapiro ED, Weibel C, et al. Association between a novel human coronavirus and Kawasaki disease. J Infect Dis 2005; 191 : 499-502.

21. Ebihara T, Endo R, Ma X, et al. Lack of association between New Haven coronavirus and Kawasaki disease. J Infect Dis 2005; 192: 351-352. 
22. Shirato K, Imada Y, Kawase M, et al. Possible involvement of infection with human coronavirus 229E, but not NL63, in Kawasaki disease. J Med Virol 2014; 86: 2146-2153.

23. Centers for Disease Control and Prevention. Multisystem inflammatory syndrome in children (MIS-C) associated with coronavirus disease 2019 (COVID-19): CDC, 2020.

24. Kim HA, Perrelli A, Ragni A, et al. Vitamin D Deficiency and the Risk of Cerebrovascular Disease. Antioxidants (Basel) 2020; 9: 327.

25. Zygmunt A, Lewiński A. Niedobór witaminy D a ciężki przebieg COVID-19 - czy istnieje związek? Medycyna Praktyczna 2020; 7-8: 69-73.

26. Regev T, Antebi M, Eytan D, et al. Pediatric Inflammatory Multisystem Syndrome With Central Nervous System Involvement and Hypocomplementemia Following SARS-COV-2 Infection. Pediatr Infect Dis J 2020; 39: e206-e207. 